TRANSACTIONS OF THE

AMERICAN MATHEMATICAL SOCIETY

Volume 361, Number 7, July 2009, Pages 3483-3500

S 0002-9947(09)04553-X

Article electronically published on February 4, 2009

\title{
ASYMPTOTICS FOR RANK PARTITION FUNCTIONS
}

\author{
KATHRIN BRINGMANN
}

\begin{abstract}
In this paper, we obtain asymptotic formulas for an infinite class of rank generating functions. As an application, we solve a conjecture of Andrews and Lewis on inequalities between certain ranks.
\end{abstract}

\section{INTRODUCTION AND STATEMENT OF RESULTS}

A partition of a positive integer $n$ is any non-increasing sequence of positive integers whose sum is $n$. As usual, let $p(n)$ denote the number of partitions of $n$. The partition function $p(n)$ has the well known infinite product generating function

$$
1+\sum_{n=1}^{\infty} p(n) q^{n}=\prod_{n=1}^{\infty} \frac{1}{1-q^{n}}=1+\sum_{n=1}^{\infty} \frac{q^{n^{2}}}{(1-q)^{2}\left(1-q^{2}\right)^{2} \cdots\left(1-q^{n}\right)^{2}} .
$$

Hardy and Ramanujan showed the following asymptotic formula for $p(n)$

$$
p(n) \sim \frac{1}{4 n \sqrt{3}} \cdot e^{\pi \sqrt{2 n / 3}} \quad(n \rightarrow \infty) .
$$

Using the modularity of the generating function for $p(n)$, Rademacher obtained an exact formula for $p(n)$. To state his result, let $I_{s}(x)$ be the usual $I$-Bessel function of order $s$, and let $e(x):=e^{2 \pi i x}$. Furthermore, if $k \geq 1$ and $n$ are integers, then let

$$
A_{k}(n):=\frac{1}{2} \sqrt{\frac{k}{12}} \sum_{\substack{x(\bmod 24 k) \\ x^{2} \equiv-24 n+1 \quad(\bmod 24 k)}} \chi_{12}(x) \cdot e\left(\frac{x}{12 k}\right),
$$

where the sum runs over the residue classes modulo $24 k$, and where

$$
\chi_{12}(x):=\left(\frac{12}{x}\right) .
$$

If $n$ is a positive integer, then Rademacher showed that

$$
p(n)=\frac{2 \pi}{(24 n-1)^{3 / 4}} \sum_{k=1}^{\infty} \frac{A_{k}(n)}{k} \cdot I_{\frac{3}{2}}\left(\frac{\pi \sqrt{24 n-1}}{6 k}\right) .
$$

The partition function also satisfies some nice congruence properties; the most famous ones are the so-called Ramanujan congruences:

$$
\begin{aligned}
p(5 n+4) \equiv 0 & (\bmod 5), \\
p(7 n+5) \equiv 0 & (\bmod 7), \\
p(11 n+6) \equiv 0 & (\bmod 11) .
\end{aligned}
$$

Received by the editors February 26, 2007.

2000 Mathematics Subject Classification. Primary 11P82, 05A17. 
In order to explain the congruences with modulus 5 and 7 combinatorially, Dyson 9] introduced the "rank" of a partition. The rank of a partition is defined to be its largest part minus the number of its parts. Dyson conjectured that the partitions of $5 n+4$ (resp. $7 n+5)$ form 5 (resp. 7) groups of equal size when sorted by their ranks modulo 5 (resp. 7). This conjecture was proved in 1954 by Atkin and Swinnerton-Dyer [5].

If $N(m, n)$ denotes the number of partitions of $n$ with rank $m$, then it is well known that

$$
R(w ; q):=1+\sum_{n=1}^{\infty} \sum_{m=-\infty}^{\infty} N(m, n) w^{m} q^{n}=1+\sum_{n=1}^{\infty} \frac{q^{n^{2}}}{(w q ; q)_{n}\left(w^{-1} q ; q\right)_{n}},
$$

where

$$
(a ; q)_{n}:=(1-a)(1-a q) \cdots\left(1-a q^{n-1}\right) .
$$

Obviously, by letting $w=1$, we obtain (1.1). Moreover, if $N_{e}(n)$ (resp. $N_{o}(n)$ ) denotes the number of partitions of $n$ with even (resp. odd) rank, then by letting $w=-1$ we obtain

$$
1+\sum_{n=1}^{\infty}\left(N_{e}(n)-N_{o}(n)\right) q^{n}=1+\sum_{n=1}^{\infty} \frac{q^{n^{2}}}{(1+q)^{2}\left(1+q^{2}\right)^{2} \cdots\left(1+q^{n}\right)^{2}} .
$$

In the following we denote this series by $f(q)$ and its $n$-th Fourier coefficient by $\alpha(n)$. The series $f(q)$ is one of the third order mock theta functions defined by Ramanujan in his last letter to Hardy dated January 1920 (see pages 127-131 of 14]). There Ramanujan claimed, without including a proof, that

$$
\alpha(n)=(-1)^{n-1} \frac{\exp \left(\pi \sqrt{\frac{n}{6}-\frac{1}{144}}\right)}{2 \sqrt{n-\frac{1}{24}}}+O\left(\frac{\exp \left(\frac{1}{2} \pi \sqrt{\frac{n}{6}-\frac{1}{144}}\right)}{\sqrt{n-\frac{1}{24}}}\right) .
$$

Dragonette [8] proved this claim in her Ph.D. thesis written in 1951 under the direction of Rademacher. Andrews [2] improved this in his Ph.D. thesis in 1964, also written under Rademacher, as

$\alpha(n)=\frac{\pi}{\sqrt{24 n-1}} \sum_{k=1}^{[\sqrt{n}]} \frac{(-1)^{\left\lfloor\frac{k+1}{2}\right\rfloor} A_{2 k}\left(n-\frac{k\left(1+(-1)^{k}\right)}{4}\right)}{k} \cdot I_{\frac{1}{2}}\left(\frac{\pi \sqrt{24 n-1}}{12 k}\right)+O\left(n^{\epsilon}\right)$.

Moreover Andrews and Dragonette made the following conjecture.

Conjecture (Andrews-Dragonette). If $n$ is a positive integer, then

$$
\alpha(n)=\frac{\pi}{(24 n-1)^{\frac{1}{4}}} \sum_{k=1}^{\infty} \frac{(-1)^{\left\lfloor\frac{k+1}{2}\right\rfloor} A_{2 k}\left(n-\frac{k\left(1+(-1)^{k}\right)}{4}\right)}{k} \cdot I_{\frac{1}{2}}\left(\frac{\pi \sqrt{24 n-1}}{12 k}\right) .
$$

In 6] Ono and the author proved this conjecture using the theory of the MaassPoincaré series. It turns out that $q^{-1} f\left(q^{24}\right)$ is the "holomorphic part" of a weak Maass form (see [7] for the definition of a weak Maass form). In [7] they showed that a similar phenomenon is true for all functions

$$
R\left(\zeta_{c}^{a} ; q\right)=: 1+\sum_{n=1}^{\infty} A\left(\frac{a}{c} ; n\right) q^{n}
$$


where $\zeta_{n}:=e^{\frac{2 \pi i}{n}}$ and $0<a<c$ are integers. More specifically, $R\left(\zeta_{c}^{a} ; q\right)$ is the holomorphic part of a weak Maass form of weight $\frac{1}{2}$. Using this deeper insight, in this paper we are able to obtain asymptotic formulas for all the coefficients $A\left(\frac{a}{c} ; n\right)$, which in turn implies asymptotics for the rank partition functions.

Before we state our result, we need some more notation. We let $k$ and $h$ be coprime integers, $h^{\prime}$ defined by $h h^{\prime} \equiv-1(\bmod k)$ if $k$ is odd and $h h^{\prime} \equiv-1(\bmod 2 k)$ if $k$ is even, $k_{1}:=\frac{k}{\operatorname{gcd}(k, c)}, c_{1}:=\frac{c}{\operatorname{gcd}(k, c)}$ and $0<l<c_{1}$ defined by the congruence $l \equiv a k_{1}\left(\bmod c_{1}\right)$. If $\frac{b}{c} \in(0,1) \backslash\left\{\frac{1}{2}, \frac{1}{6}, \frac{5}{6}\right\}$, then define the integer $s(b, c)$ by

$$
s(b, c):= \begin{cases}0 & \text { if } 0<\frac{b}{c}<\frac{1}{6} \\ 1 & \text { if } \frac{1}{6}<\frac{b}{c}<\frac{1}{2} \\ 2 & \text { if } \frac{1}{2}<\frac{b}{c}<\frac{5}{6} \\ 3 & \text { if } \frac{5}{6}<\frac{b}{c}<1\end{cases}
$$

In particular, set $s:=s\left(l, c_{1}\right)$. Let $\omega_{h, k}$ be the multiplier occuring in the transformation law of the partition function $p(n)$. This is explicitly given by

$$
\omega_{h, k}:=\exp (\pi i t(h, k))
$$

where

$$
t(h, k):=\sum_{\mu}\left(\left(\frac{\mu}{k}\right)\right)\left(\left(\frac{h \mu}{k}\right)\right)
$$

Here

$$
((x)):= \begin{cases}x-\lfloor x\rfloor-\frac{1}{2} & \text { if } x \in \mathbb{R} \backslash \mathbb{Z} \\ 0 & \text { if } x \in \mathbb{Z}\end{cases}
$$

Moreover we define, for $n, m \in \mathbb{Z}$, the following sums of Kloosterman type:

$$
B_{a, c, k}(n, m):=(-1)^{a k+1} \sin \left(\frac{\pi a}{c}\right) \sum_{h \bmod k^{*}} \frac{\omega_{h, k}}{\sin \left(\frac{\pi a h^{\prime}}{c}\right)} \cdot e^{-\frac{3 \pi i a^{2} k_{1} h^{\prime}}{c}} \cdot e^{\frac{2 \pi i}{k}\left(n h+m h^{\prime}\right)}
$$

if $c \mid k$, and

$$
D_{a, c, k}(n, m):=(-1)^{a k+l} \sum_{h \bmod k^{*}} \omega_{h, k} \cdot e^{\frac{2 \pi i}{k}\left(n h+m h^{\prime}\right)} .
$$

Here the sums run through all primitive residue classes modulo $k$. Moreover, for $c \nmid k$, let

$$
\delta_{c, k, r}:= \begin{cases}-\left(\frac{1}{2}+r\right) \frac{l}{c_{1}}+\frac{3}{2}\left(\frac{l}{c_{1}}\right)^{2}+\frac{1}{24} & \text { if } 0<\frac{l}{c_{1}}<\frac{1}{6} \\ -\frac{5 l}{2 c_{1}}+\frac{3}{2}\left(\frac{l}{c_{1}}\right)^{2}+\frac{25}{24}-r\left(1-\frac{l}{c_{1}}\right) & \text { if } \frac{5}{6}<\frac{l}{c_{1}}<1 \\ 0 & \text { otherwise }\end{cases}
$$

and for $0<\frac{l}{c_{1}}<\frac{1}{6}$ or $\frac{5}{6}<\frac{l}{c_{1}}<1$

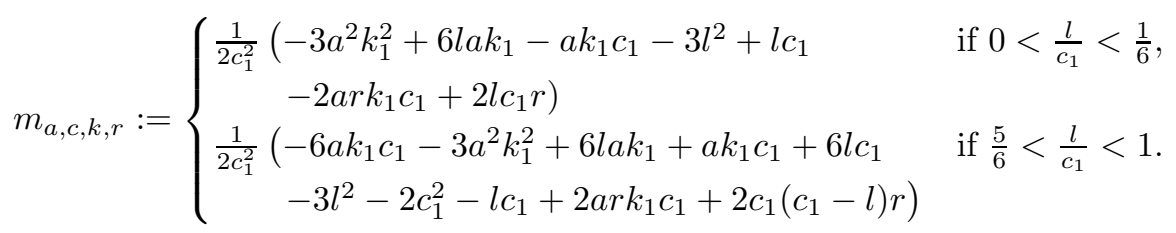

Remark. It is not hard to see that $m_{a, c, k, r} \in \mathbb{Z}$. 
We obtain the following asymptotic formulas for the coefficients $A\left(\frac{a}{c} ; n\right)$.

Theorem 1.1. If $0<a<c$ are coprime integers and $c$ is odd, then for positive integers $n$ we have that

$$
\begin{gathered}
A\left(\frac{a}{c} ; n\right)=\frac{4 \sqrt{3} i}{\sqrt{24 n-1}} \sum_{\substack{1 \leq k \leq \sqrt{n} \\
c \mid k}} \frac{B_{a, c, k}(-n, 0)}{\sqrt{k}} \cdot \sinh \left(\frac{\pi \sqrt{24 n-1}}{6 k}\right) \\
+\frac{8 \sqrt{3} \cdot \sin \left(\frac{\pi a}{c}\right)}{\sqrt{24 n-1}} \sum_{\substack{1 \leq k \leq \sqrt{n} \\
c \neq k \\
c \geq 0 \\
c, k, r}} \frac{D_{a, c, k}\left(-n, m_{a, c, k, r}\right)}{\sqrt{k}} \cdot \sinh \left(\frac{\pi \sqrt{2 \delta_{c, k, r}(24 n-1)}}{\sqrt{3} k}\right)+O_{c}\left(n^{\epsilon}\right) .
\end{gathered}
$$

Four remarks. 1) One can easily see that the second sum is empty for $c \in\{3,5\}$.

2) For a fixed choice of $a$ and $c$, we can in principle obtain exact formulas for $A\left(\frac{a}{c} ; n\right)$ by modifying an argument given in [6] to prove (1.8).

3) Similarly as in this paper, one can also prove asymptotic formulas for generalized ranks as defined in [11. Since the proof is basically the same, we do not give it here.

4) One could also generalize our results to the case that $c$ is even, but for simplicity we restrict ourselves to the case where $c$ is odd.

If we denote by $N(a, c ; n)$ the number of partitions of $n$ with rank congruent $a$ $(\bmod c)$, then it is easy to conclude the following:

Corollary 1.2. For integers $0 \leq a<c$, where $c$ is an odd integer, we have

$$
\begin{aligned}
N(a, c ; n) & =\frac{2 \pi}{c \cdot \sqrt{24 n-1}} \sum_{k=1}^{\infty} \frac{A_{k}(n)}{k} \cdot I_{\frac{3}{2}}\left(\frac{\pi \sqrt{24 n-1}}{6 k}\right) \\
& +\frac{1}{c} \sum_{j=1}^{c-1} \zeta_{c}^{-a j}\left(\frac{4 \sqrt{3} i}{\sqrt{24 n-1}} \sum_{c_{j} \mid k} \frac{B_{j^{\prime}, c_{j}, k}(-n, 0)}{\sqrt{k}} \sinh \left(\frac{\pi}{6 k} \sqrt{24 n-1}\right)\right. \\
+\frac{8 \sqrt{3} \sin \left(\frac{\pi j}{c}\right)}{\sqrt{24 n-1}} & \left.\sum_{\substack{k, r \\
c_{j}+k \\
\delta_{j^{\prime}, c, k, r}>0}} \frac{D_{j^{\prime}, c_{j}, k}\left(-n, m_{j^{\prime}, c_{j}, k, r}\right)}{\sqrt{k}} \sinh \left(\sqrt{\frac{2 \delta_{j^{\prime}, c_{j}, k, r}(24 n-1)}{3}} \frac{\pi}{k}\right)\right)+O_{c}\left(n^{\epsilon}\right),
\end{aligned}
$$

where $c_{j}=\frac{c}{\operatorname{gcd}\left(c_{j}\right)}$ and $j^{\prime}=\frac{j}{\operatorname{gcd}\left(c_{j}\right)}$.

This corollary implies some conjectures of Andrews and Lewis. In [4, 13, they showed

$$
\begin{aligned}
N(0,2 ; 2 n) & <N(1,2 ; 2 n) & & \text { if } n \geq 1, \\
N(0,4 ; n) & >N(2,4 ; n) & & \text { if } 26<n \equiv 0,1 \quad(\bmod 4), \\
N(0,4 ; n) & <N(2,4 ; n) & & \text { if } 26<n \equiv 2,3 \quad(\bmod 4) .
\end{aligned}
$$

Moreover, they conjectured (see Conjecture 1 of [4]) the following.

Conjecture (Andrews and Lewis). For all $n>0$, we have

$$
\begin{array}{ll}
N(0,3 ; n)<N(1,3 ; n) & \text { if } n \equiv 0 \text { or } 2 \quad(\bmod 3), \\
N(0,3 ; n)>N(1,3 ; n) & \text { if } n \equiv 1 \quad(\bmod 3) .
\end{array}
$$

A careful analysis of Corollary 1.2 gives the following theorem. 
Theorem 1.3. The Andrews-Lewis Conjecture is true for all $n \notin\{3,9,21\}$ in which case we have equality in (1.14).

Remark. From Corollary 1.2 we see that

$$
N(0,3 ; n)-N(1,3 ; n) \sim-\frac{8 \sin \left(\frac{\pi}{18}-\frac{2 \pi n}{3}\right) \sinh \left(\frac{\pi \cdot \sqrt{24 n-1}}{18}\right)}{\sqrt{24 n-1}} .
$$

This directly implies the Andrews-Lewis Conjecture for $n$ sufficiently large $n$. For example for $n=1200$ we have

$$
N(0,3 ; 1200)-N(1,3 ; 1200)=-29873238925,
$$

whereas (1.15) gives

$$
-29873204830.34 \text {. }
$$

The paper is organized as follows: In Section 2 we prove a transformation law for the functions $R\left(\zeta_{c}^{a} ; q\right)$. The behavior under the generators of $\mathrm{SL}_{2}(\mathbb{Z})$ was also studied in $[12$ and in $[7$. However here we prove a more general result since we need the occurring roots of unity and integrals explicitly for every element in $\mathrm{SL}_{2}(\mathbb{Z})$. In Section 4 we prove Theorem 1.1 and Corollary 1.2 by using the Circle Method. For this we need some estimates shown in Section 3 . Section 5 is dedicated to the proof of Theorem 1.3 .

\section{Modular transformation formulas}

In this section we prove a transformation law for the functions $R\left(\zeta_{c}^{a} ; q\right)$. For this define

$$
N\left(\frac{a}{c} ; q\right):=\frac{1}{(q ; q)_{\infty}}\left(1+\sum_{n=1}^{\infty} \frac{(-1)^{n}\left(1+q^{n}\right)\left(2-2 \cos \left(\frac{2 \pi a}{c}\right)\right)}{1-2 q^{n} \cos \left(\frac{2 \pi a}{c}\right)+q^{2 n}} \cdot q^{\frac{n(3 n+1)}{2}}\right)
$$

In [12] it is shown that

$$
R\left(\zeta_{c}^{a} ; q\right)=N\left(\frac{a}{c} ; q\right)
$$

Moreover let

$$
\begin{aligned}
N(a, b, c ; q):=\frac{i}{2(q ; q)_{\infty}}\left(\sum_{m=0}^{\infty} \frac{(-1)^{m} e^{-\frac{\pi i a}{c}} \cdot q^{\frac{m}{2}(3 m+1)+m s(b, c)+\frac{b}{2 c}}}{1-e^{-\frac{2 \pi i a}{c}} \cdot q^{m+\frac{b}{c}}}\right. \\
\left.-\sum_{m=1}^{\infty} \frac{(-1)^{m} e^{\frac{\pi i a}{c}} \cdot q^{\frac{m}{2}(3 m+1)-m s(b, c)-\frac{b}{2 c}}}{1-e^{\frac{2 \pi i a}{c}} \cdot q^{m-\frac{b}{c}}}\right) .
\end{aligned}
$$

Remark. It is easy to see that the above definition coincides with the definition, given in [7].

For each $\nu \in \mathbb{Z}$ define

$$
\begin{aligned}
H_{a, c}(x) & :=\frac{\cosh (x)}{\sinh \left(x+\frac{\pi i a}{c}\right) \cdot \sinh \left(x-\frac{\pi i a}{c}\right)} \\
I_{a, c, k, \nu}(z) & :=\int_{\mathbb{R}} e^{-\frac{3 \pi z x^{2}}{k}} \cdot H_{a, c}\left(\frac{\pi i \nu}{k}-\frac{\pi i}{6 k}-\frac{\pi z x}{k}\right) d x .
\end{aligned}
$$


It is easy to see that

$$
\begin{aligned}
H_{a, c}(-x) & =H_{a, c}(x), \\
H_{a, c}(x) & =\frac{2(1+q) \cdot q^{\frac{1}{2}}}{\left(1-2 \cos \left(\frac{2 \pi a}{c}\right) q+q^{2}\right)}
\end{aligned}
$$

for $q=e^{2 x}$. We show the following transformation law for the function $N\left(\frac{a}{c} ; q\right)$.

Theorem 2.1. We assume the same notation as in the Introduction. Moreover, let $z \in \mathbb{C}$ with $\operatorname{Re}(z)>0, q:=e^{\frac{2 \pi i}{k}(h+i z)}$, and $q_{1}:=e^{\frac{2 \pi i}{k}\left(h^{\prime}+\frac{i}{z}\right)}$.

(1) If $c \mid k$, then

$$
\begin{aligned}
N\left(\frac{a}{c} ; q\right)= & \frac{(-1)^{a k+1} i \sin \left(\frac{\pi a}{c}\right) \cdot \omega_{h, k}}{\sin \left(\frac{\pi a h^{\prime}}{c}\right) z^{\frac{1}{2}}} \cdot e^{-\frac{3 \pi i a^{2} k_{1} h^{\prime}}{c}} \cdot e^{\frac{\pi}{12 k}\left(z^{-1}-z\right)} \cdot N\left(\frac{a h^{\prime}}{c} ; q_{1}\right) \\
& +\frac{2 \sin ^{2}\left(\frac{\pi a}{c}\right) \cdot \omega_{h, k}}{k} e^{-\frac{\pi z}{12 k}} \cdot z^{\frac{1}{2}} \sum_{\nu \bmod k}(-1)^{\nu} e^{-\frac{3 \pi i h^{\prime} \nu^{2}}{k}+\frac{\pi i h^{\prime} \nu}{k}} \cdot I_{a, c, k, \nu}(z) .
\end{aligned}
$$

(2) If $c \nmid k$, then

$$
\begin{gathered}
N\left(\frac{a}{c} ; q\right)=\frac{4 i(-1)^{a k+l+1} \cdot \sin \left(\frac{\pi a}{c}\right) \omega_{h, k}}{z^{\frac{1}{2}}} \cdot e^{-\frac{2 \pi i h^{\prime} s a}{c}-\frac{3 \pi i a^{2} h^{\prime} k_{1}}{c c_{1}}+\frac{6 \pi i h^{\prime} l a}{c c_{1}}} \\
\cdot q_{1}^{\frac{s l}{c_{1}}-\frac{3 l^{2}}{2 c_{1}^{2}}} \cdot e^{\frac{\pi}{12 k}\left(z^{-1}-z\right)} \times N\left(a h^{\prime}, \frac{l c}{c_{1}}, c ; q_{1}\right) \\
+\frac{2 \sin ^{2}\left(\frac{\pi a}{c}\right) \omega_{h, k}}{k} e^{-\frac{\pi z}{12 k}} \cdot z^{\frac{1}{2}} \sum_{\nu}(-1)^{\nu} e^{-\frac{3 \pi i h^{\prime} \nu^{2}}{k}+\frac{\pi i h^{\prime} \nu}{k}} I_{a, c, k, \nu}(z) .
\end{gathered}
$$

Proof. We modify the proof of [2]. We easily see, using $1-\cos (2 x)=2 \sin (x)^{2}$, that

$$
(q ; q)_{\infty} \cdot N\left(\frac{a}{c} ; q\right)=\sin ^{2}\left(\frac{\pi a}{c}\right) \sum_{n \in \mathbb{Z}}(-1)^{n} H_{a, c}\left(\frac{\pi i n}{k}(h+i z)\right) \cdot e^{\frac{3 \pi i(h+i z) n^{2}}{k}}
$$

Writing $n=k m+\nu$ with $0 \leq \nu<k, m \in \mathbb{Z}$, and using the fact that $(h, k)=1$, gives us that (2.2) equals

$$
\sin ^{2}\left(\frac{\pi a}{c}\right) \sum_{\nu=0}^{k-1}(-1)^{\nu} e^{\frac{3 \pi i h \nu}{k}} \sum_{m \in \mathbb{Z}}(-1)^{m} H_{a, c}\left(\frac{\pi i h \nu}{k}-\frac{\pi(k m+\nu) z}{k}\right) \cdot e^{-\frac{3 \pi z(k m+\nu)^{2}}{k}} .
$$

Using Poisson summation and substituting $x \mapsto k x+\nu$ gives us that the inner sum equals

$$
\frac{1}{k} \sum_{n \in \mathbb{Z}} \int_{\mathbb{R}} H_{a, c}\left(\frac{\pi i h \nu}{k}-\frac{\pi x z}{k}\right) \cdot e^{\frac{\pi i(2 n+1)(x-\nu)}{k}-\frac{3 \pi z x^{2}}{k}} d x .
$$

Strictly speaking for $c \mid k$ there may be a pole at $x=0$. In this case we take the principal part of the integral. Inserting (2.4) into (2.3) we see that the summation only depends on $\nu(\bmod k)$. Moreover, by changing $\nu$ into $-\nu, x$ into $-x$, and $n$ 
into $-(n+1)$, we see that the sum over $n$ with $n \leq-1$ equals the sum with $n \geq 0$. Thus (2.3) equals

$$
\begin{array}{r}
\frac{2 \sin ^{2}\left(\frac{\pi a}{c}\right)}{k} \sum_{\nu}(-1)^{\nu} e^{\frac{3 \pi i h \nu}{k}} \sum_{n \in \mathbb{N}} \int_{\mathbb{R}} H_{a, c}\left(\frac{\pi i h \nu}{k}-\frac{\pi x z}{k}\right) \\
\cdot e^{\frac{\pi i(2 n+1)(x-\nu)}{k}-\frac{3 \pi z x^{2}}{k}} d x .
\end{array}
$$

To see where the poles of the integrant lie, we introduce the function

$$
S_{a, c, k}(x):=\frac{\sinh \left(c_{1} x\right)}{\sinh \left(\frac{x}{k}+\frac{\pi i a}{c}\right) \cdot \sinh \left(\frac{x}{k}-\frac{\pi i a}{c}\right)}
$$

which is entire as a function of $x$. Using that $c$ is odd we can write the integrand in (2.5) as

$$
\frac{(-1)^{h \nu} \cosh \left(\frac{\pi i h \nu}{k}-\frac{\pi x z}{k}\right) \cdot e^{\frac{\pi i(2 n+1)(x-\nu)}{k}-\frac{3 \pi z x^{2}}{k}} \cdot S_{a, c, k}(\pi x z-\pi i h \nu)}{\sinh \left(\pi c_{1} x z\right)} .
$$

From this we see that only the poles can lie in the points

$$
x_{m}:=\frac{i m}{c_{1} z} \quad(m \in \mathbb{Z}) .
$$

We treat the cases with $c \mid k$ or $c \nmid k$ seperately.

If $c \mid k$, then $c_{1}=1$. One computes that each choice \pm leads at most for one $\nu$ $(\bmod k)$ to a non-zero residue, and that this $\nu$ can be chosen as

$$
\nu_{m}^{ \pm}:=-h^{\prime}\left(m- \pm a k_{1}\right) .
$$

We denote the corresponding residues by $\lambda_{n, m}^{ \pm}$. By shifting the path of integration through the points

$$
\omega_{n}:=\frac{(2 n+1) i}{6 z},
$$

we have to take those points $x_{m}$ into account for which $n \geq 3 m \geq 0$. Setting $r_{0}:=\frac{1}{2}$ and $r_{m}:=1$ for $m \in \mathbb{N}$, we obtain by the Residue Theorem

$$
(q ; q)_{\infty} \cdot N\left(\frac{a}{c} ; q\right)=\sum_{11}+\sum_{2}
$$

where

$$
\begin{aligned}
\sum_{11}:= & \frac{4 \pi i \sin ^{2}\left(\frac{\pi a}{c}\right)}{k} \sum_{m \geq 0} r_{m}(-1)^{\nu_{m}^{ \pm}} e^{\frac{3 \pi i h\left(\nu_{m}^{ \pm}\right)^{2}}{k}} \sum_{n=3 m}^{\infty} \lambda_{n, m}^{ \pm}, \\
\sum_{2}:= & \frac{2 \sin ^{2}\left(\frac{\pi a}{c}\right)}{k} \sum_{(\bmod k)}(-1)^{\nu} e^{\frac{3 \pi i h \nu^{2}}{k}} \\
& \times \sum_{n \in \mathbb{N}} \int_{-\infty+\omega_{n}}^{\infty+\omega_{n}} H_{a, c}\left(\frac{\pi i h \nu}{k}-\frac{\pi x z}{k}\right) \cdot e^{\frac{\pi i(2 n+1)(x-\nu)}{k}-\frac{3 \pi x^{2} z}{k}} d x .
\end{aligned}
$$

If $c \nmid k$, then a pole can only occur if $m \equiv \pm a k_{1}\left(\bmod c_{1}\right)$. Writing $c_{1} m \pm l$ instead of $m$ with $m \geq 0$ for the choice + and $m>0$ for the choice - and $l$ as in the 
Introduction, we see that to each choice there corresponds exactly one $\nu(\bmod k)$ and we can choose $\nu$ as

$$
\nu_{m}^{ \pm}:=-h^{\prime}\left(m \pm \frac{1}{c_{1}}\left(l-a k_{1}\right)\right) .
$$

As before, we denote the corresponding residues by $\lambda_{n, m}^{ \pm}$. By shifting the path of integration through the points $\omega_{n}$, we have to take those points $x_{m}$ into account for which $\frac{2 n+1}{6}>\frac{c_{1} m \pm l}{c_{1}}$. One can see that this is equivalent to

$$
n \geq 3 m \pm s,
$$

where $s$ was defined in (1.9). By the Residue Theorem we obtain

$$
(q ; q)_{\infty} \cdot N\left(\frac{a}{c} ; q\right)=\sum_{12}+\sum_{2},
$$

where $\sum_{2}$ is given as before and $\sum_{12}$ is defined as

$$
\begin{aligned}
\frac{4 \pi i \sin ^{2}\left(\frac{\pi a}{c}\right)}{k}\left(\sum_{m \geq 0}(-1)^{\nu_{m}^{+}} e^{\frac{3 \pi i h\left(\nu_{m}^{+}\right)^{2}}{k}} \sum_{n=3 m+s}^{\infty} \lambda_{n, m}^{+}\right. & \\
& \left.+\sum_{m \geq 1}(-1)^{\nu_{m}^{-}} e^{\frac{3 \pi i h\left(\nu_{m}^{-}\right)^{2}}{k}} \sum_{n=3 m-s}^{\infty} \lambda_{n, m}^{-}\right) .
\end{aligned}
$$

We first consider the sums $\sum_{11}$ and $\sum_{12}$. We have

$$
\lambda_{n, m}^{ \pm}=-\frac{k \cdot \cosh \left(\frac{\pi i h \nu_{m}^{ \pm}}{k}-\frac{\pi x_{m} z}{k}\right) \cdot e^{\frac{\pi i(2 n+1)\left(x_{m}-\nu_{m}^{ \pm}\right)}{k}-\frac{3 \pi z x_{m}^{2}}{k}}}{\pi z \cdot \cosh \left(\frac{\pi i h \nu_{m}^{ \pm}}{k}-\frac{\pi x_{m} z}{k} \pm \frac{\pi i a}{c}\right) \cdot \sinh \left(\frac{\pi i h \nu_{m}^{ \pm}}{k}-\frac{\pi x_{m} z}{k}-\mp \frac{\pi i a}{c}\right)} .
$$

From this one directly sees that

$$
\lambda_{n+1, m}^{ \pm}=\exp \left(\frac{2 \pi i}{k}\left(x_{m}-\nu_{m}^{ \pm}\right)\right) \cdot \lambda_{n, m}^{ \pm} .
$$

Thus

$$
\sum_{n=3 m+r}^{\infty} \lambda_{n, m}^{ \pm}=\frac{\lambda_{3 m+r, m}^{ \pm}}{1-\exp \left(\frac{2 \pi i}{k}\left(x_{m}-\nu_{m}^{ \pm}\right)\right)} \quad(r \in\{0, \pm s\}) .
$$

A straightforward but lenghty calculation gives

$$
\begin{aligned}
\sum_{11}= & \frac{(-1)^{a k+1} i \sin \left(\frac{\pi a}{c}\right)}{\sin \left(\frac{\pi a h^{\prime}}{c}\right) z} \cdot e^{-\frac{3 \pi i a^{2} k_{1} h^{\prime}}{c}} \cdot\left(q_{1} ; q_{1}\right)_{\infty} \cdot N\left(\frac{a h^{\prime}}{c} ; q_{1}\right), \\
\sum_{12}= & \frac{4 i \sin \left(\frac{\pi a}{c}\right)(-1)^{a k+l+1}}{z} e^{-\frac{2 \pi i h^{\prime} s a}{c}-\frac{3 \pi i h^{\prime} a^{2} k_{1}}{c c_{1}}+\frac{6 \pi i h^{\prime} l a}{c c_{1}}} q_{1}^{\frac{s l}{c_{1}}-\frac{3}{2}\left(\frac{l}{c_{1}}\right)^{2}} \\
& \cdot\left(q_{1} ; q_{1}\right)_{\infty} N\left(h^{\prime} a, \frac{l c}{c_{1}}, c ; q_{1}\right) .
\end{aligned}
$$

Here we have to be a little careful since for $c \mid k(c \nmid k)$ we only have that $h \nu$ is congruent to $m \mp a k_{1}\left(m \pm \frac{1}{c_{1}}\left(l-a k_{1}\right)\right)$ modulo $k$ but not necessarily modulo $2 k$ if $k$ is odd. 
We next turn to the computation of $\sum_{2}$. With the same argument as before we can change the sum over $\mathbb{N}$ into a sum over $\mathbb{Z}$. Making the translation $x \mapsto x+\omega_{n}$ and writing $n=3 p+\delta$ with $p \in \mathbb{Z}$ and $\delta \in\{0, \pm 1\}$ gives us that

$$
\begin{aligned}
& \sum_{2}=\frac{\sin ^{2}\left(\frac{\pi a}{c}\right)}{k} \sum_{\nu} \sum_{(\bmod k)}(-1)^{\nu} e^{\frac{3 \pi i h \nu^{2}}{k}} \sum_{\substack{p \in \mathbb{Z} \\
\delta \in\{0, \pm 1\}}} e^{-\frac{\pi(6 p+2 \delta+1)^{2}}{12 k z}-\frac{\pi i \nu(6 p+2 \delta+1)}{k}} \\
& \cdot \int_{-\infty}^{\infty} H_{a, c}\left(\frac{\pi i h \nu}{k}-\frac{\pi i(6 p+2 \delta+1)}{6 k}-\frac{\pi z x}{k}\right) \cdot e^{-\frac{3 \pi z x^{2}}{k}} d x .
\end{aligned}
$$

Now $\left(h^{\prime}, k\right)=1$ implies that $-h^{\prime}(\nu+p)$ runs modulo $k$ if $\nu$ does. Thus we can change $\nu$ into $-h^{\prime}(\nu+p)$ which leads to

$$
\begin{aligned}
& \sum_{2}=\frac{\sin ^{2}\left(\frac{\pi a}{c}\right)}{k} \sum_{\substack { \nu \\
\begin{subarray}{c}{(\bmod k) \\
\delta \in\{0 \pm 1\} \\
p \in \mathbb{Z}\}{ \nu \\
\begin{subarray} { c } { ( \operatorname { m o d } k ) \\
\delta \in \{ 0 \pm 1 \} \\
p \in \mathbb { Z } \} } }\end{subarray}}(-1)^{\nu+p} q_{1}^{\frac{p}{2}(3 p+2 \delta+1)} \cdot e^{-\frac{\pi(2 \delta+1)^{2}}{12 k z}+\frac{\pi i h^{\prime}\left(-3 \nu^{2}+(2 \delta+1) \nu\right)}{k}} \\
& \cdot \int_{-\infty}^{\infty} H_{a, c}\left(\frac{\pi i \nu}{k}-\frac{\pi i(2 \delta+1)}{6 k}-\frac{\pi z x}{k}\right) \cdot e^{-\frac{3 \pi z x^{2}}{k}} d x
\end{aligned}
$$

Now the integral is independent of $p$ and the sum over $p$ equals

$$
\sum_{p \in \mathbb{Z}}(-1)^{p} \cdot q_{1}^{\frac{1}{2} p(3 p+(2 \delta+1))} .
$$

If $\delta=1$, then (2.7) vanishes since the $p$-th and the $-(p+1)$-th term cancel. Changing $\nu$ into $-\nu, p$ into $-p$ and $x$ into $-x$, we see that the terms in (2.6) corresponding to $\delta=-1$ and $\delta=0$ are equal. Moreover in this case (2.7) equals $\left(q_{1} ; q_{1}\right)_{\infty}$. Thus

$$
\sum_{2}=\frac{2 \sin ^{2}\left(\frac{\pi a}{c}\right) \cdot\left(q_{1} ; q_{1}\right)_{\infty} \cdot e^{-\frac{\pi}{12 k z}}}{k} \sum_{\nu}(-1)^{\nu} e^{-\frac{3 \pi i h^{\prime} \nu^{2}}{k}+\frac{\pi i h^{\prime} \nu}{k}} \cdot I_{a, c, k, \nu}(z) .
$$

Now the theorem follows easily using the transformation law

$$
\left(q_{1} ; q_{1}\right)_{\infty}=\omega_{h, k} \cdot z^{\frac{1}{2}} \cdot e^{\frac{\pi}{12 k}\left(z^{-1}-z\right)} \cdot(q ; q)_{\infty} .
$$

\section{Some estimates}

In this section we estimate the function $I_{a, c, k, \nu}(z)$, defined in Section 2 and some Kloosterman sums.

Lemma 3.1. Assume that $n \in \mathbb{N}, \nu \in \mathbb{Z}, z:=\frac{k}{n}-k \Phi i,-\frac{1}{k\left(k+k_{1}\right)} \leq \Phi \leq \frac{1}{k\left(k+k_{2}\right)}$, where $\frac{h_{1}}{k_{1}}<\frac{h}{k}<\frac{h_{2}}{k_{2}}$ are adjacent Farey fractions in the Farey sequence of order $N$, with $N:=\left\lfloor n^{\frac{1}{2}}\right\rfloor$. Then

$$
z^{\frac{1}{2}} \cdot I_{a, c, k, \nu}(z) \ll k \cdot n^{\frac{1}{4}} \cdot g_{a, c, k, \nu},
$$

where $g_{a, c, k, \nu},:=\left(\min \left(6 k c\left\{\frac{\nu}{k}-\frac{1}{6 k}+\frac{a}{c}\right\}, 6 k c\left\{\frac{\nu}{k}-\frac{1}{6 k}-\frac{a}{c}\right\}\right)\right)^{-1}$, where $\{x\}:=$ $x-\lfloor x\rfloor$ for $x \in \mathbb{R}$. Here the implied constant is independent of $a, k$, and $\nu$.

Proof. We write $\frac{\pi z}{k}=C e^{i A}$ with $C>0$. Then $|A|<\frac{\pi}{2}$ since $\operatorname{Re}(z)>0$. Making the substitution $\tau=\frac{\pi z x}{k}$ gives

$$
z^{\frac{1}{2}} \cdot I_{a, c, k, \nu}(z)=\frac{k}{\pi z^{\frac{1}{2}}} \int_{S} e^{-\frac{3 k \tau^{2}}{\pi z}} \cdot H_{a, c}\left(\frac{\pi i \nu}{k}-\frac{\pi i}{6 k}-\tau\right) d \tau,
$$


where $\tau$ runs on the ray through 0 of elements with argument $\pm A$. One can see that for $0 \leq t \leq A$

$$
\left|e^{-\frac{3 k R^{2} e^{2 i t}}{\pi z}} \cdot H_{a, c}\left(\frac{\pi i \nu}{k}-\frac{\pi i}{6 k} \pm R e^{i t}\right)\right| \rightarrow 0 \quad(R \rightarrow \infty) .
$$

Moreover, since $c$ is odd, the integrant in (3.1) can only have poles in points $i r$ with $r \in \mathbb{R} \backslash\{0\}$. Thus we can shift the path of integration to the real line and get

$$
z^{\frac{1}{2}} \cdot I_{a, c, k, \nu}(z)=\frac{k}{\pi z^{\frac{1}{2}}} \int_{\mathbb{R}} e^{-\frac{3 k t^{2}}{\pi z}} \cdot H_{a, c}\left(\frac{\pi i \nu}{k}-\frac{\pi i}{6 k}-t\right) d t .
$$

We can show the following estimates:

$$
\begin{gathered}
\mid e^{-\frac{3 k t^{2}}{\pi z} \mid}=e^{-\frac{3 k}{\pi} \operatorname{Re}\left(\frac{1}{z}\right) t^{2}}, \\
\left|\cosh \left(\frac{\pi i \nu}{k}-\frac{\pi i}{6 k}-t\right)\right| \leq e^{t}, \\
\left|\sinh \left(\frac{\pi i \nu}{k}-\frac{\pi i}{6 k}-t \pm \frac{\pi i a}{c}\right)\right| \geq \begin{cases}\frac{e^{t}}{2 \sqrt{2}} & \text { if } t \geq 1, \\
\left|\sin \left(\frac{\pi \nu}{k}-\frac{\pi}{6 k} \pm \frac{\pi a}{c}\right)\right| & \text { if } t \leq 1,\end{cases} \\
\qquad \sin \left(\frac{\pi \nu}{k}-\frac{\pi}{6 k}+\frac{\pi a}{c}\right)|| \sin \left(\frac{\pi \nu}{k}-\frac{\pi}{6 k}-\frac{\pi a}{c}\right) \mid \\
\left.\left.\left.\qquad \frac{\nu}{k}-\frac{1}{6 k}+\frac{a}{c}\right\},\left\{\frac{\nu}{k}-\frac{1}{6 k}-\frac{a}{c}\right\}\right)\right) .
\end{gathered}
$$

Thus

$$
z^{\frac{1}{2}} \cdot I_{a, c, k, \nu}(z) \ll \frac{k}{\left(\min \left(\left\{\frac{\nu}{k}-\frac{1}{6 k}+\frac{a}{c}\right\},\left\{\frac{\nu}{k}-\frac{1}{6 k}-\frac{a}{c}\right\}\right)\right)|z|^{\frac{1}{2}}} \int_{\mathbb{R}} e^{-\frac{3 k}{\pi} t^{2} \operatorname{Re}\left(\frac{1}{z}\right)} d t .
$$

Making the substitution $t \mapsto \sqrt{\frac{3 k \operatorname{Re}\left(\frac{1}{z}\right)}{\pi}} \cdot t$ and using the estimate

$$
\operatorname{Re}\left(\frac{1}{z}\right)^{-\frac{1}{2}} \cdot|z|^{-\frac{1}{2}} \leq 2^{\frac{1}{4}} \cdot n^{\frac{1}{4}} \cdot k^{-\frac{1}{2}}
$$

gives the lemma.

We next estimate certain sums of Kloosterman type.

Lemma 3.2. Let $n, m \in \mathbb{Z}, 0 \leq \sigma_{1}<\sigma_{2} \leq k, D \in \mathbb{Z}$ with $(D, k)=1$.

(1) We have

$$
\sum_{\substack{h(\bmod k)^{*} \\ \sigma_{1} \leq D h^{\prime} \leq \sigma_{2}}} \omega_{h, k} \cdot e^{\frac{2 \pi i}{k}\left(h n+h^{\prime} m\right)} \ll \operatorname{gcd}(24 n+1, k)^{\frac{1}{2}} \cdot k^{\frac{1}{2}+\epsilon} .
$$

(2) If $c \mid k$, then we have

$$
\begin{aligned}
& (-1)^{a k+1} \sin \left(\frac{\pi a}{c}\right) \sum_{\substack{h \\
\sigma_{1} \leq D h^{\prime} \leq \sigma_{2}}} \frac{\omega_{h, k}}{\sin \left(\frac{\pi a h^{\prime}}{c}\right)} \cdot e^{-\frac{3 \pi i a^{2} k_{1} h^{\prime}}{c}} \cdot e^{\frac{2 \pi i}{k}\left(h n+h^{\prime} m\right)} \\
& \ll \operatorname{gcd}(24 n+1, k)^{\frac{1}{2}} \cdot k^{\frac{1}{2}+\epsilon}
\end{aligned}
$$

where the implied constants are independent of $a$ and $k$. 
Proof. Equation (3.3) is basically proven in 2]; thus we only consider (3.4). We set $\tilde{c}:=c$ if $k$ is odd and $\tilde{c}:=2 c$ if $k$ is even. Clearly $\frac{e^{-\frac{3 \pi i a^{2} k_{1} h^{\prime}}{c}}}{\sin \left(\frac{\pi a h^{\prime}}{c}\right)}$ only depends on the residue class of $h^{\prime}(\bmod \tilde{c})$. Thus we can write (3.4) as

$$
(-1)^{a k+1} \sin \left(\frac{\pi a}{c}\right) \sum_{c_{j}} \frac{e^{-\frac{3 \pi i a^{2} k_{1} c_{j}}{c}}}{\sin \left(\frac{\pi a c_{j}}{c}\right)} \sum_{\substack{\left.h \\ \sigma_{1} \leq D h^{\prime} \leq\right)_{2} \\ h^{\prime} \equiv c_{j}(\bmod \tilde{c})}} \omega_{h, k} \cdot e^{\frac{2 \pi i}{k}\left(h n+h^{\prime} m\right)}
$$

where $c_{j}$ runs through a set of primitive residues $(\bmod \tilde{c})$. The inner sum can be rewritten as

$$
\begin{aligned}
& \frac{1}{\tilde{c}} \sum_{\substack{h \\
\sigma_{1} \leq D h^{\prime} \leq \sigma_{2}}} \omega_{h, k} \cdot e^{\frac{2 \pi i}{k}\left(h n+h^{\prime} m\right)} \sum_{r} e_{(\bmod \tilde{c})} e^{\frac{2 \pi i r}{\tilde{c}}\left(h^{\prime}-c_{j}\right)} \\
& =\frac{1}{\tilde{c}} \sum_{r} e^{-\frac{2 \pi i r c_{j}}{\tilde{c}}} \sum_{\substack{h \\
(\bmod \tilde{c}) \\
\sigma_{1} \leq D h^{\prime} \leq \sigma_{2}}} \omega_{h, k} \cdot e^{\frac{2 \pi i}{k}\left(h n+h^{\prime}\left(m+\frac{k r}{\tilde{c}}\right)\right)} .
\end{aligned}
$$

Using (3.3) now easily gives (3.4).

Remark. If $a=1$ and $c=3$, then it is not hard to see that the left hand side of (3.4) simplifies to

$$
\sum_{\substack{h \\ \sigma_{1} \leq D h^{\prime} \leq \sigma_{2}}}\left(\frac{h}{3}\right) \cdot \omega_{h, k} \cdot e^{\frac{2 \pi i}{k}\left(h n+h^{\prime} m\right)} .
$$

\section{Proof of Theorem 1.1}

Proof of Theorem 1.1. For the proof of Theorem 1.1 we use the Hardy-Ramanujan method. By Cauchy's Theorem we have for $n>0$

$$
A\left(\frac{a}{c} ; n\right)=\frac{1}{2 \pi i} \int_{C} \frac{N\left(\frac{a}{c} ; q\right)}{q^{n+1}} d q
$$

where $C$ is an arbitrary path inside the unit circle surrounding 0 counterclockwise. Choosing the circle with radius $e^{-\frac{2 \pi}{n}}$ and as a parametrisation $q=e^{-\frac{2 \pi}{n}+2 \pi i t}$ with $0 \leq t \leq 1$ gives us

$$
A\left(\frac{a}{c} ; n\right)=\int_{0}^{1} N\left(\frac{a}{c} ; e^{-\frac{2 \pi}{n}+2 \pi i t}\right) \cdot e^{2 \pi-2 \pi i n t} d t .
$$

Define

$$
\vartheta_{h, k}^{\prime}:=\frac{1}{k\left(k_{1}+k\right)}, \quad \vartheta_{h, k}^{\prime \prime}:=\frac{1}{k\left(k_{2}+k\right)},
$$

where $\frac{h_{1}}{k_{1}}<\frac{h}{k}<\frac{h_{2}}{k_{2}}$ are adjacent Farey fractions in the Farey sequence of order $N:=\left\lfloor n^{1 / 2}\right\rfloor$. From the theory of Farey fractions it is known that

$$
\frac{1}{k+k_{j}} \leq \frac{1}{N+1} \quad(j=1,2) \text {. }
$$


We decompose the path of integration in paths along the Farey $\operatorname{arcs}-\vartheta_{h, k}^{\prime} \leq \Phi \leq$ $\vartheta_{h, k}^{\prime \prime}$, where $\Phi=t-\frac{h}{k}$ and $0 \leq h \leq k \leq N$ with $(h, k)=1$. Thus

$$
A\left(\frac{a}{c} ; n\right)=\sum_{h, k} e^{-\frac{2 \pi i h n}{k}} \int_{-\vartheta_{h, k}^{\prime}}^{\vartheta_{h, k}^{\prime \prime}} N\left(\frac{a}{c} ; e^{\frac{2 \pi i}{k}(h+i z)}\right) \cdot e^{\frac{2 \pi n z}{k}} d \Phi
$$

where $z=\frac{k}{n}-k \Phi i$. Applying Theorem 2.1 gives

$$
\begin{aligned}
& A\left(\frac{a}{c} ; n\right)=i \sin \left(\frac{\pi a}{c}\right) \sum_{\substack{h, k \\
c \mid k}} \omega_{h, k} \frac{(-1)^{a k+1}}{\sin \left(\frac{\pi a h^{\prime}}{c}\right)} \\
& \cdot e^{-\frac{3 \pi i a^{2} k_{1} h^{\prime}}{c}-\frac{2 \pi i h n}{k}} \int_{-\vartheta_{h, k}^{\prime}}^{\vartheta_{h, k}^{\prime \prime}} z^{-\frac{1}{2}} \cdot e^{\frac{2 \pi z}{k}\left(n-\frac{1}{24}\right)+\frac{\pi}{12 k z}} \\
& \times N\left(\frac{a h^{\prime}}{c} ; q_{1}\right) d \Phi-4 i \sin \left(\frac{\pi a}{c}\right) \sum_{\substack{h, k \\
c \nmid k}} \omega_{h, k}(-1)^{a k+l} \\
& \cdot e^{-\frac{2 \pi i h^{\prime} s a}{c}-\frac{3 \pi i h^{\prime} a^{2} k_{1}}{c c_{1}}+\frac{6 \pi i h^{\prime} l a}{c c_{1}}-\frac{2 \pi i h n}{k}} \\
& \cdot \int_{-\vartheta_{h, k}^{\prime}}^{\vartheta_{h, k}^{\prime \prime}} z^{-\frac{1}{2}} \cdot e^{\frac{2 \pi z}{k}\left(n-\frac{1}{24}\right)+\frac{\pi}{12 k z}} \cdot q_{1}^{\frac{s l}{c_{1}}-\frac{3 l^{2}}{2 c_{1}^{2}}} \\
& \cdot N\left(a h^{\prime}, \frac{l c}{c_{1}}, c ; q_{1}\right) d \Phi+2 \sin ^{2}\left(\frac{\pi a}{c}\right) \sum_{h, k} \frac{\omega_{h, k}}{k} \cdot e^{-\frac{2 \pi i h n}{k}} \\
& \cdot \sum_{\nu}(-1)^{\nu} e^{-\frac{3 \pi i h^{\prime} \nu^{2}}{k}+\frac{\pi i h^{\prime} \nu}{k}} \int_{-\vartheta_{h, k}^{\prime}}^{\vartheta_{h, k}^{\prime \prime}} e^{\frac{2 \pi z}{k}\left(n-\frac{1}{24}\right)} \\
& \cdot z^{\frac{1}{2}} \cdot I_{a, c, k, \nu}(z) d \Phi=: \sum_{1}+\sum_{2}+\sum_{3} .
\end{aligned}
$$

To estimate $\sum_{1}$, we write

$$
N\left(\frac{a h^{\prime}}{c} ; q_{1}\right)=: 1+\sum_{r \in \mathbb{N}} a(r) \cdot e^{\frac{2 \pi i m_{r} h^{\prime}}{k}} \cdot e^{-\frac{2 \pi r}{k z}}
$$

where $m_{r}$ is a sequence in $\mathbb{Z}$ and the coefficients $a(r)$ are independent of $a, c, k$, and $h$. We treat the constant term and the term coming from $r \geq 1$ seperately, since they contribute to the main term and to the error term, respectively. We denote the associated sums by $S_{1}$ and $S_{2}$, respectively, and first estimate $S_{2}$. Throughout we need the easily verified fact that $\operatorname{Re}(z)=\frac{k}{n}, \operatorname{Re}\left(\frac{1}{z}\right)>\frac{k}{2},|z|^{-\frac{1}{2}} \leq n^{\frac{1}{2}} \cdot k^{-\frac{1}{2}}$, and $\vartheta_{h, k}^{\prime}+\vartheta_{h, k}^{\prime \prime} \leq \frac{2}{k(N+1)}$. Since $k_{1}, k_{2} \leq N$, we can write

$$
\int_{-\vartheta_{h, k}^{\prime}}^{\vartheta_{h, k}^{\prime \prime}}=\int_{-\frac{1}{k(N+k)}}^{\frac{1}{k(N+k)}}+\int_{-\frac{1}{k\left(k_{1}+k\right)}}^{-\frac{1}{k(N+k)}}+\int_{\frac{1}{k(N+k)}}^{\frac{1}{k\left(k_{2}+k\right)}}
$$

and denote the associated sums by $S_{21}, S_{22}$, and $S_{23}$, respectively. 
We first consider $S_{21}$. Using Lemma 3.2 gives us

$$
\begin{aligned}
S_{21} \ll \mid \sum_{r=1}^{\infty} a(r) \sum_{c \mid k}(-1)^{a k+1} \sin \left(\frac{\pi a}{c}\right) \sum_{h} \frac{\omega_{h, k}}{\sin \left(\frac{\pi a h^{\prime}}{c}\right)} \cdot e^{-\frac{3 \pi i a^{2} k_{1} h^{\prime}}{c}-\frac{2 \pi i h n}{k}+\frac{2 \pi i m_{r} h^{\prime}}{k}} \\
\quad \int_{-\frac{1}{k(N+k)}}^{\frac{1}{k(N+k)}} z^{-\frac{1}{2}} \cdot e^{-\frac{2 \pi}{k z}\left(r-\frac{1}{24}\right)+\frac{2 \pi z}{k}\left(n-\frac{1}{24}\right)} d \Phi \mid \\
\ll \sum_{r=1}^{\infty}|a(r)| \cdot e^{-\pi r} \sum_{k} k^{-1+\epsilon} \cdot(24 n-1, k)^{\frac{1}{2}} \\
\ll \sum_{\substack{d \mid(24 n-1) \\
d \leq N}} d^{\frac{1}{2}} \sum_{k \leq \frac{N}{d}}(d k)^{-1+\epsilon} \ll n^{\epsilon} \sum_{d \mid(24 n-1)} d^{-\frac{1}{2}} \ll n^{\epsilon} .
\end{aligned}
$$

Since $S_{22}$ and $S_{23}$ are treated in exactly the same way we only consider $S_{22}$. Writing

$$
\int_{-\frac{1}{k\left(k+k_{1}\right)}}^{-\frac{1}{k(N+k)}}=\sum_{l=k_{1}+k}^{N+k-1} \int_{-\frac{1}{k l}}^{-\frac{1}{k(l+1)}},
$$

we see

$$
\begin{aligned}
& S_{22} \ll \mid \sum_{r=1}^{\infty} a(r) \sum_{c \mid k} \sum_{l=N+1}^{N+k-1} \int_{-\frac{1}{k l}}^{-\frac{1}{k(l+1)}} z^{-\frac{1}{2}} \cdot e^{-\frac{2 \pi}{k z}\left(r-\frac{1}{24}\right)+\frac{2 \pi z}{k}\left(n-\frac{1}{24}\right)} d \Phi \\
&(-1)^{a k+1} \sin \left(\frac{\pi a}{c}\right) \sum_{\substack{h \\
N<k+k_{1} \leq l}} \frac{\omega_{h, k}}{\sin \left(\frac{\pi a h^{\prime}}{c}\right)} \cdot e^{-\frac{3 \pi i a^{2} k_{1} h^{\prime}}{c}} \cdot e^{-\frac{2 \pi i h n}{k}} e^{\frac{2 \pi i m_{r} h^{\prime}}{k}} \mid .
\end{aligned}
$$

It follows from the theory of Farey fractions that

$$
\begin{array}{r}
k_{1} \equiv-h^{\prime} \quad(\bmod k), \quad k_{2} \equiv h^{\prime} \quad(\bmod k), \\
N-k<k_{1} \leq N, \quad N-k<k_{2} \leq N .
\end{array}
$$

Thus we can use Lemma 3.2 and estimate (4.3) as in the case of $S_{21}$. Therefore $\sum_{1}$ equals

$$
\begin{aligned}
i \sin \left(\frac{\pi a}{c}\right) \sum_{\substack{h, k \\
c \mid k}} \omega_{h, k} \cdot \frac{(-1)^{a k+1}}{\sin \left(\frac{\pi a h^{\prime}}{c}\right)} \cdot e^{-\frac{3 \pi i a^{2} k_{1} h^{\prime}}{c}-\frac{2 \pi i h n}{k}} \int_{-\vartheta_{h, k}^{\prime}}^{\vartheta_{h, k}^{\prime \prime}} z^{-\frac{1}{2}} \\
\cdot e^{\frac{2 \pi z}{k}\left(n-\frac{1}{24}\right)+\frac{\pi}{12 k z}} d \Phi+O\left(n^{\epsilon}\right) .
\end{aligned}
$$

The sum $\sum_{2}$ is treated in a similar manner; we make some comments about necessary modifications. One can show that one can write

$$
\begin{aligned}
e^{-\frac{2 \pi i h^{\prime} s a}{c}-\frac{3 \pi i h^{\prime} a^{2} k_{1}}{c c_{1}}+\frac{6 \pi i h^{\prime} l a}{c c_{1}}+\frac{\pi}{12 k z}} \cdot q_{1}^{\frac{s l}{c_{1}}-\frac{3 l^{2}}{2 c_{1}^{2}}} \cdot N & \left(a h^{\prime}, \frac{l c}{c_{1}}, c ; q_{1}\right) \\
& =: \sum_{r \geq r_{0}} b(r) e^{\frac{2 \pi i m_{r} h^{\prime}}{k}} e^{-\frac{\pi i r}{12 k c^{2} z}},
\end{aligned}
$$

where $m_{r}$ is a sequence in $\mathbb{Z}, r_{0} \in \mathbb{Z}$. A lengthy but straightforward calculation shows that the terms with $r$ negative, i.e., the the part that gives a contribution 
for the main term, can only arise if $s \in\{0,3\}$. If $s=0$, then the contribution is given by

$$
\frac{i}{2} e^{-\frac{3 \pi i h^{\prime} a^{2} k_{1}}{c c_{1}}+\frac{6 \pi i h^{\prime} l a}{c c_{1}}-\frac{\pi i a h^{\prime}}{c}+\frac{\pi}{12 k z}} \cdot q_{1}^{-\frac{3 l^{2}}{2 c_{1}^{2}}+\frac{l}{2 c_{1}}} \sum_{\substack{r \\ \delta_{c, k, r}>0}} e\left(-\frac{a h^{\prime} r}{c}\right) \cdot q_{1}^{\frac{l r}{c_{1}}} .
$$

If $s=3$, then the contribution is given by

$$
\frac{i}{2} e^{-\frac{6 \pi i a h^{\prime} a}{c}-\frac{3 \pi i h^{\prime} a^{2} k_{1}}{c c_{1}}+\frac{6 \pi i h^{\prime} l a}{c c_{1}}+\frac{\pi i a h^{\prime}}{c}+\frac{\pi}{12 k z}} \cdot q_{1}^{\frac{5 l}{2 c_{1}}-\frac{3 l^{2}}{2 c_{1}^{2}}-1} \sum_{\substack{r \\ \delta_{c, k, r}>0}} e\left(\frac{a h^{\prime} r}{c}\right) \cdot q_{1}^{\left(1-\frac{l}{c_{1}}\right) r} .
$$

One can write the sum over $k$ in groups in which the $k$ 's have the same values for $c_{1}$ and $l$ and thus the same values for $\delta_{c, k, r}$, where $\delta_{c, k, r}$ was defined in (1.13). In each class the condition $\delta_{c, k, r}>0$ is now independent of the $k$ in this class; in every group there are only finitely many terms with $\delta_{c, k, r}>0$, and the number is bounded in terms of $c$. Moreover the coefficient $b(r)$ is independent of $k$ and $a$ in a fixed class. Now the terms with negative exponents can be estimated as before; thus $\sum_{2}$ equals

$$
\begin{aligned}
2 \sin \left(\frac{\pi a}{c}\right) \sum_{\substack{k, r \\
c \nmid k \\
\delta_{c, k, r}>0}}(-1)^{a k+l} \sum_{h} \omega_{h, k} e^{\frac{2 \pi i}{k}\left(-n h+m_{a, c, k, r} h^{\prime}\right)} \\
\cdot \int_{-\vartheta_{h, k}^{\prime}}^{\vartheta_{h, k}^{\prime \prime}} z^{-\frac{1}{2}} e^{\frac{2 \pi z}{k}\left(n-\frac{1}{24}\right)+\frac{2 \pi}{k z} \delta_{c, k, r}} d \Phi+O\left(n^{\epsilon}\right) .
\end{aligned}
$$

We turn back to the estimation of $\sum_{1}$. We can write

$$
\int_{-\vartheta_{h, k}^{\prime}}^{\vartheta_{h, k}^{\prime \prime}}=\int_{-\frac{1}{k N}}^{\frac{1}{k N}}-\int_{-\frac{1}{k N}}^{-\frac{1}{k\left(k+k_{1}\right)}}-\int_{\frac{1}{k\left(k+k_{2}\right)}}^{\frac{1}{k N}}
$$

and denote the associated sums by $S_{11}, S_{12}$, and $S_{13}$, respectively. The sums $S_{12}$ and $S_{13}$ contribute to the error terms. Since they have the same shape, we only consider $S_{12}$. Writing

$$
\int_{-\frac{1}{k N}}^{-\frac{1}{k\left(k+k_{1}\right)}}=\sum_{l=N}^{k+k_{1}-1} \int_{-\frac{1}{k l}}^{-\frac{1}{k(l+1)}}
$$

gives

$$
\begin{aligned}
S_{12} \ll \sum_{c \mid k} & \sum_{l=N}^{N+k-1} \int_{-\frac{1}{k l}}^{-\frac{1}{k(l+1)}} z^{-\frac{1}{2}} e^{\frac{\pi}{12 k z}+\frac{2 \pi z}{k}\left(n-\frac{1}{24}\right)} d \Phi \\
& (-1)^{a k+1} \sin \left(\frac{\pi a}{c}\right) \sum_{\substack{h \\
l<k+k_{1}-1 \leq N+k-1}} \frac{\omega_{h, k}}{\sin \left(\frac{\pi a h^{\prime}}{c}\right)} \cdot e^{-\frac{3 \pi i a^{2} k_{1} h^{\prime}}{c}} \cdot e^{-\frac{2 \pi i h n}{k}} .
\end{aligned}
$$

Using $\operatorname{Re}(z)=\frac{k}{n}, \operatorname{Re}\left(\frac{1}{z}\right)<k$, and $|z|^{2} \geq \frac{k^{2}}{n^{2}}$, this sum can be estimated as before against $O\left(n^{\epsilon}\right)$. Thus, using (1.11),

$$
\sum_{1}=i \sum_{c \mid k} B_{a, c, k}(-n, 0) \int_{-\frac{1}{k N}}^{\frac{1}{k N}} z^{-\frac{1}{2}} \cdot e^{\frac{2 \pi z}{k}\left(n-\frac{1}{24}\right)+\frac{\pi}{12 k z}} d \Phi+O\left(n^{\epsilon}\right) .
$$


In the same way, we obtain, using (1.12),

$$
\begin{aligned}
& \sum_{2}=2 \sin \left(\frac{\pi a}{c}\right) \sum_{\substack{k, r \\
c+k \\
\delta_{c, k, r}>0}} D_{a, c, k}\left(-n, m_{a, c, k, r}\right) \int_{-\frac{1}{k N}}^{\frac{1}{k N}} z^{-\frac{1}{2}} \\
& \cdot e^{\frac{2 \pi z}{k}\left(n-\frac{1}{24}\right)+\frac{2 \pi}{k z} \delta_{c, k, r}} d \Phi+O\left(n^{\epsilon}\right) .
\end{aligned}
$$

To finish the estimation of $\sum_{1}$ and $\sum_{2}$ we have to consider integrals of the form

$$
I_{k, r}:=\int_{-\frac{1}{k N}}^{\frac{1}{k N}} z^{-\frac{1}{2}} \cdot e^{\frac{2 \pi}{k}\left(z\left(n-\frac{1}{24}\right)+\frac{r}{z}\right)} d \Phi .
$$

Substituting $z=\frac{k}{n}-i k \Phi$ gives

$$
I_{k, r}=\frac{1}{k i} \int_{\frac{k}{n}-\frac{i}{N}}^{\frac{k}{n}+\frac{i}{N}} z^{-\frac{1}{2}} \cdot e^{\frac{2 \pi}{k}\left(z\left(n-\frac{1}{24}\right)+\frac{r}{z}\right)} d z .
$$

We now denote the circle through $\frac{k}{n} \pm \frac{i}{N}$ and tangent to the imaginary axis at 0 by $\Gamma$. If $z=x+i y$, then $\Gamma$ is given by $x^{2}+y^{2}=\alpha x$, with $\alpha=\frac{k}{n}+\frac{n}{N^{2} k}$. Using the fact that $2>\alpha>\frac{1}{k}, \operatorname{Re}(z) \leq \frac{k}{n}$, and $\operatorname{Re}\left(\frac{1}{z}\right)<k$ on the smaller arc, we can show that the integral along the smaller arc is in $O\left(n^{-\frac{3}{4}}\right)$. Moreover the path of integration in (4.5) can be changed by Cauchy's Theorem into the larger arc of $\Gamma$. Thus

$$
I_{k, r}=\frac{1}{k i} \int_{\Gamma} z^{-\frac{1}{2}} \cdot e^{\frac{2 \pi}{k}\left(z\left(n-\frac{1}{24}\right)+\frac{r}{z}\right)} d z+O\left(n^{-\frac{1}{8}}\right) .
$$

Making the substitution $t=\frac{2 \pi r}{k z}$ gives

$$
I_{k, r}=\frac{2 \pi}{k}\left(\frac{2 \pi r}{k}\right)^{\frac{1}{2}} \frac{1}{2 \pi i} \int_{\gamma-i \infty}^{\gamma+i \infty} t^{-\frac{3}{2}} \cdot e^{t+\frac{\alpha}{t}} d t+O\left(n^{-\frac{1}{8}}\right),
$$

where $\gamma \in \mathbb{R}$ and $\alpha=\frac{\pi^{2} r}{6 k^{2}}(24 n-1)$. By the Hankel integral formula we now get

$$
I_{k, r}=\frac{4 \sqrt{3}}{\sqrt{k(24 n-1)}} \sinh \left(\sqrt{\frac{2 r(24 n-1)}{3}} \frac{\pi}{k}\right)+O\left(n^{-\frac{1}{8}}\right) .
$$

Thus we obtain, estimating the Kloosterman sums with the same arguments as before and using Lemma 3.1 ,

$$
\begin{gathered}
\sum_{1}+\sum_{2}=\frac{4 \sqrt{3} i}{\sqrt{24 n-1}} \sum_{c \mid k} \frac{B_{a, c, k}(-n, 0)}{\sqrt{k}} \cdot \sinh \left(\frac{\pi}{6 k} \sqrt{24 n-1}\right) \\
+\frac{8 \sqrt{3} \sin \left(\frac{\pi a}{c}\right)}{\sqrt{24 n-1}} \sum_{\substack{k, r \\
c \nmid k \\
\delta_{c, k, r}>0}} \frac{D_{a, c, k}\left(-n, m_{a, c, k, r}\right)}{\sqrt{k}} \cdot \sinh \left(\sqrt{\frac{2 \delta_{c, k, r}(24 n-1)}{3}} \frac{\pi}{k}\right)+O\left(n^{\epsilon}\right) .
\end{gathered}
$$

To estimate $\sum_{3}$, we split the path of integration as in (4.2) and estimate the sums in the same way as before, using the fact that

$$
\sum_{1 \leq \nu \leq k} g_{a, c, k, \nu} \ll \sum_{1 \leq \nu \leq 6 c k} \frac{1}{\nu} \ll k^{\epsilon} .
$$


Proof of Corollary 1.2, Corollary 1.2 follows directly from the identity

$$
\sum_{n=0}^{\infty} N(a, c ; n) q^{n}=\frac{1}{c} \sum_{n=0}^{\infty} p(n) q^{n}+\frac{1}{c} \sum_{j=1}^{c-1} \zeta_{c}^{-a j} \cdot R\left(\zeta_{c}^{j} ; q\right)
$$

and from (1.4).

\section{Proof of Theorem 1.3}

Here we prove the Andrews-Lewis Conjecture. Using equation (4.6) and

$$
N\left(\frac{1}{3} ; q\right)=N\left(\frac{2}{3} ; q\right)
$$

easily gives us that

$$
N(0,3 ; n)<N(1,3 ; n) \Leftrightarrow A\left(\frac{1}{3} ; n\right)<0 .
$$

Thus Theorem 2 follows from the following.

Proposition 5.1. We have for all $n \notin\{1,3,7\}$

$$
\begin{aligned}
A\left(\frac{1}{3} ; 3 n\right) & <0, \\
A\left(\frac{1}{3} ; 3 n+1\right) & >0, \\
A\left(\frac{1}{3} ; 3 n+2\right) & <0 .
\end{aligned}
$$

Remark. For $n \in\{1,3,7\}$, equations (5.2) and (5.3) still hold, and in (5.1) we have equality.

Proof. With MAPLE we compute that the proposition holds for $n \leq 3000$. Thus in the following, we may assume that $n>3000$. Actually we only need $n \geq 300$.

We first consider the main term given by

$$
\frac{4 \sqrt{3} i}{(24 n-1)^{\frac{1}{2}}} \sum_{3 \mid k} \frac{B_{1,3, k}(-n, 0)}{\sqrt{k}} \cdot \sinh \left(\frac{\pi}{6 k} \sqrt{24 n-1}\right) .
$$

Using definition (1.10) it is easy to see that

$$
B_{1,3,3}(-n, 0)=2 i \sin \left(\frac{\pi}{18}-\frac{2 \pi n}{3}\right) .
$$

Thus the term corresponding to $k=3$ is given by

$$
-\frac{8 \sin \left(\frac{\pi}{18}-\frac{2 \pi n}{3}\right) \cdot \sinh \left(\frac{\pi}{18} \sqrt{24 n-1}\right)}{(24 n-1)^{\frac{1}{2}}} .
$$

Using the trivial bound for Kloosterman sums gives us the fact that the remaining terms can be estimated against

$$
\frac{12}{(24 n-1)^{\frac{1}{2}}} \sum_{2 \leq k \leq \frac{N}{3}} k^{\frac{1}{2}} \cdot \sinh \left(\frac{\pi}{18 k} \sqrt{24 n-1}\right) .
$$

Next we make the $O$-term in Theorem 1.1 explicit. For this we use the same notation as in the proof of Theorem 1.1. Instead of using Lemma 3.2, we estimate 
the Kloosterman sums trivially. The contribution coming from $N\left(\frac{h^{\prime}}{3} ; q_{1}\right)$ can be estimated against

$$
\frac{2 \cdot e^{2 \pi+\frac{\pi}{24}}}{\sqrt{3}} \sum_{r=1}^{\infty}|a(r)| \cdot e^{-\pi r} \sum_{1 \leq k \leq \frac{N}{3}} k^{-\frac{1}{2}} .
$$

Computing the first few coefficients and then using the fact that for $n>1$

$$
p(n)<\exp \left(\pi \sqrt{\frac{2 n}{3}}\right)
$$

easily gives us

$$
\sum_{r=1}^{\infty}|a(r)| \cdot e^{-\pi r} \leq 0.06
$$

In the same way we can estimate the contribution coming from $N\left(h^{\prime}, l, 3 ; q_{1}\right)$ by

$$
4 \sqrt{3} \cdot e^{2 \pi} \sum_{r=1}^{\infty}|b(r)| \cdot e^{-\frac{\pi r}{144}} \sum_{\substack{1 \leq k \leq N \\ 3 \nmid k}} k^{-\frac{1}{2}} .
$$

Moreover, distinguishing the cases $l=1$ and $l=2$, we get

$$
\sum_{r=1}^{\infty}|b(r)| \cdot e^{-\frac{\pi r}{216}} \leq 0.353
$$

By making the path of integration symmetric, we introduce an error that can be estimated against

$$
2 \sqrt{3} \cdot e^{2 \pi+\frac{\pi}{12}} \cdot n^{-\frac{1}{2}} \sum_{1 \leq k \leq \frac{N}{3}} k^{\frac{1}{2}}
$$

Integrating along the smaller arc of $\Gamma$ gives an error that can be estimated against

$$
8 \pi \cdot e^{2 \pi+\frac{\pi}{24}} \cdot n^{-\frac{3}{4}} \sum_{1 \leq k \leq \frac{N}{3}} k
$$

Moreover the estimate in Lemma 3.1 can be made explicit as

$$
2^{\frac{5}{4}} \cdot \frac{e+e^{-1}}{3} \cdot n^{\frac{1}{4}}\left(\min \left(\left\{\frac{\nu}{k}-\frac{1}{6 k}+\frac{1}{3}\right\},\left\{\frac{\nu}{k}-\frac{1}{6 k}+\frac{1}{3}\right\}\right)\right)^{-1} .
$$

This easily gives

$$
\begin{aligned}
\sum_{3} \leq 2^{\frac{1}{4}} \cdot\left(e+e^{-1}\right) \cdot e^{2 \pi} & \\
\cdot & n^{-\frac{1}{4}} \sum_{k} \frac{1}{k} \sum_{\nu=1}^{k}\left(\min \left(\left\{\frac{\nu}{k}-\frac{1}{6 k}+\frac{1}{3}\right\},\left\{\frac{\nu}{k}-\frac{1}{6 k}-\frac{1}{3}\right\}\right)\right)^{-1} .
\end{aligned}
$$

Combining all errors one can show that the term in (5.4) is dominant for $n \geq$ 300 .

\section{ACKNOWLEDGEMENTS}

The author thanks K. Ono, B. Kane, and F. Garvan for helpful comments on an earlier version of the paper. 


\section{REFERENCES}

[1] G. E. Andrews, The theory of partitions, Cambridge Univ. Press, Cambridge, 1998. MR:1634067 (99c:11126)

[2] G. E. Andrews, On the theorems of Watson and Dragonette for Ramanujan's mock theta functions, Amer. J. Math. 88 No. 2 (1966), pages 454-490. MR.0200258 (34:157)

[3] G. E. Andrews, Mock theta functions, Theta functions - Bowdoin 1987, Part 2 (Brunswick, ME, 1987), pages 283-297, Proc. Sympos. Pure Math. 49, Part 2, Amer. Math. Soc., Providence, RI, 1989. MR1013178 (90h:33005)

[4] G. E. Andrews and R. P. Lewis, The ranks and cranks of partitions moduli 2, 3 and 4, J. Number Th. 85 (2000), pages 74-84. MR 1800302 (2001k:11200)

[5] A. O. L. Atkin and H. P. F. Swinnerton-Dyer, Some properties of partitions, Proc. London Math. Soc. 66 No. 4 (1954), pages 84-106. MR0060535 (15:685d)

[6] K. Bringmann and K. Ono, The $f(q)$ mock theta function conjecture and partition ranks, Invent. Math. 165 (2006), pages 243-266. MR2231957 (2007e:11127)

[7] K. Bringmann and K. Ono, Dysons ranks and Maass forms, Annals, accepted for publication.

[8] L. Dragonette, Some asymptotic formulae for the mock theta series of Ramanujan, Trans. Amer. Math. Soc. 72 No. 3 (1952), pages 474-500. MR0049927 (14:248g)

[9] F. Dyson, Some guesses in the theory of partitions, Eureka (Cambridge) 8 (1944), pages 10-15.

[10] F. Dyson, A walk through Ramanujan's garden, Ramanujan revisited (Urbana-Champaign, Ill. 1987), Academic Press, Boston, 1988, pages 7-28. MR938957 (89f:01065)

[11] F. Garvan, Generalization of Dyson's rank and non-Rogers-Ramanujan partitions, Manuscripta Math. 84 (1994), pages 343-359. MR1291125 (95e:11115)

[12] B. Gordon and R. McIntosh, Modular transformations of Ramanujan's fifth and seventh order mock theta functions, Ramanujan J. 7 (2003), pages 193-222. MR2035802 (2005a:11020)

[13] R. P. Lewis, The ranks of partitions modulo 2, Discuss. Math. 167/168 (1997), pages 445449. MR.1446763 (98d:11122)

[14] S. Ramanujan, The lost notebook and other unpublished papers, Narosa, New Delhi, 1988. MR 947735 (89j:01078)

Mathematisches Institut, Universtät Köln, Weyertal 86-90, 50931 Köln, Germany

E-mail address: kbringma@math.uni-koeln.de 